\title{
Inter- vs. intraspecific genetic variability of morphologically similar Ligophorus species
}

\author{
Ekaterina Vodiasova \\ IBSS RAS, Sevastopol, Russia \\ eavodiasova@gmail.com \\ Alexei Ermolenko \\ FSC the East Asia Terrestrial \\ Biodiversity, Vladivistok, Russia \\ ermolenko_alexey@mail.ru
}

\author{
Evgenija Dmitrieva \\ IBSS RAS, Sevastopol, Russia \\ genijadmitrieva@gmail.com \\ Dmitry Atopkin \\ FSC the East Asia Terrestrial \\ Biodiversity, Vladivistok, Russia \\ pan2006_82@mail.ru
}

\author{
Olga Shikhat \\ IBSS RAS, Sevastopol, Russia \\ shixatolya@yandex.ru
}

\begin{abstract}
Monogeneans of Ligophorus (Platyhelminthes), specific parasites of the fish of Mugilidae, present comprises 60 species based mainly on morphology. The contradictions between morphological and genetic (rDNA fragments) inter- vs. intraspecific variability is shown for some species. Six species of Ligophorus newly collected in the Black Sea, South China Sea and Sea of Japan and 80 sequences of ITS1 and 285 were obtained. Two species, $L$. satunensis and $L$. fenestrum, from the three neighboring regions of the Western Pacific (WP) have no morphological differences but diverge from each other in 3.3$5.8 \%$ by $28 \mathrm{~S}$ rRNA. In contrast to this, the low intraspecific genetic variability $(0.1-0.6 \%$ for $28 S)$ is revealed for sympatric $L$. pilengas and L. llewellyni, distinguishing in morphology. Due to the different rates of divergence of species of this genus, the integrated morphological and genetic approach, considering the ecology, phylogeography and isolation of host populations, for the correct phylogenetic reconstruction of this genus are suggested.
\end{abstract}

Keywords - molecular taxonomy, Ligophorus, phylogeny, morphology, rDNA

\section{Motivation and aim}

The traditional taxonomy of Ligophorus spp. is based on the morphology of the structures of the posterior attachment organ and male reproductive system. Among dozens of articles dedicating systematics of these monogeneans, only five of them uses rDNA sequences to identify phylogenetic relationships between species. However, a lack of enough data on intraspecific genetic variation leads sometimes to results contradicting with morphology. The most species of Ligophorus have only two or three rDNA sequences deposited to GenBank. Moreover, the genetic distances between species varies widely, from 0.4 to $50.2 \%$ for both ITS1 and 28S [13].

The aim of this work is to analyze the interspecific and intraspecific genetic variability of Ligophorus species with similar morphology.

\section{Methods}

Six species of Ligophorus newly collected in the Black Sea, South China Sea and Sea of Japan and additional data on some species from GenBank NCBI are included in analysis. 80 sequences of ITS1 and $28 \mathrm{~S}$ from 40 specimens were obtained. DNA extraction was carried out using innuPREP DNA Mini Kit. The fragment of 28S was amplified using the primers U178 (5'- GCA CCC GCT GAA YTT AAG -3'; [4]) and LSU1200R (5'- GCA TAG TTC ACC ATC TTT CGG 3'; [5]). ITS1 was amplified with the primers Lig18endF (5GTC TTG CGG TTC ACG CTG CT -3) and Lig5.8R (5- GAT
ACT CGA GCC GAG TGA TCC -3) [1]. The reaction conditions consisted of initial denaturation at $95^{\circ} \mathrm{C}$ for $3 \mathrm{~min}$, followed by 35 cycles of denaturation at $94^{\circ} \mathrm{C}$ for $40 \mathrm{~s}$, annealing at $56^{\circ} \mathrm{C}$ for $30 \mathrm{~s}$ and extension at $72^{\circ} \mathrm{C}$ for $45 \mathrm{~s}$, the final extension at $72^{\circ} \mathrm{C}$ for $4 \mathrm{~min}$. PCR products were sequenced in both directions using the standard BigDye Terminator Cycle Sequencing Ready Reaction Kit and a ABI PRISM 3130 analyzer (Applied Biosystems Inc.). The obtained fragments of rDNA were aligned in the BioEdit software program [71. Evolutionary analyses were conducted in MEGA7 [8] using a specimen of Ergenstrema mugilis from GenBank as a reference sequence for ITS1 (GenBank JN996835) and 28S (GenBank JN996800).

\section{Results}

Two species, L. satunensis and L. fenestrum, from the three neighboring regions of the Western Pacific (WP) have no morphological differences but diverge from each other in $3.3-5.8 \%$ by $28 \mathrm{~S}$ rRNA. Additionally, the high intraspecific genetic variability in this gene is revealed for L. johorensis $(2.6 \%)$ and L. kedahensis $(2.8 \%)$ also from different localitis of WP. The same level of genetic divergence $(2.7 \%)$ was previously observed between two morphologically very similar sympatric species, L. uruguayense and L. saladensis [3]. However, inter- and intraspecific distances overlap for $28 \mathrm{~S}$ for $L$. saladensis and $L$. mediterraneus from different hosts and regions. The low intraspecific genetic variability $(0.1-0.6 \%$ for $28 \mathrm{~S})$ is also revealed for sympatric $L$. pilengas and L. llewellyni, distinguishing in morphology.

The revealed contradictions between morphological and genetic variability indicate different rates of divergence of species of this genus. Thus, a comprehensive morphological and genetic study, considering the ecology, phylogeography and isolation of host populations, is necessary for the correct phylogenetic reconstruction of Ligophorus.

\section{Acknowledgment}

This work is funded by a scientific theme of IBSS number AAAA-A18-118020890074-2, RFBR and Sevastopol project number 20-44-920004, and "Ecolan 3.1" of Vietnam-Russian Tropical Research and Technological Centre.

\section{References}

[1] Blasco-Costa I., Miguez-Lozano R., Sarabeev V., Balbuena J.A. (2012) Molecular phylogeny of species of Ligophorus (Monogenea: Dactylogyridae) and their affinities within the Dactylogyridae. Parasitology International. 61: 619-627.

[2] Pakdee W, Ogawa K, Pornruseetriratn S, Thaenkham U, Yeemin T (2018). The first record of Ligophorus Euzet \& Suriano, 1977 (Monogenea: Dactylogyridae) on Crenimugil buchanani 
(Teleostei: Muglidae) from Thailand based on morphological and molecular analyses. Journal of Helminthology. 93(6):752-762.

[3] Marchiori N.C., Pariselle A., Pereira Jr. J., Agnèse J.-F., Durand J.-D., Vanhove M.P.M. 2015: A comparative study of Ligophorus uruguayense and $L$. saladensis (Monogenea: Ancyrocephalidae) from Mugil liza (Teleostei: Mugilidae) in southern Brazil. Folia Parasitol. 62: 024. doi: 10.14411/fp.2015.024

[4] Lockyer AE, Olson PD, Littlewood DTJ. Utility of complete large and small subunit rRNA genes in resolving the phylogeny of the Neodermata (Platyhelminthes): im- plications and a review of the cercomer theory. Biological Journal of the Linnean Society $2003 ; 78: 155-71$.
[5] Littlewood DTJ, Curini-Galletti M, Herniou EA. The interrelationships of Proseriata (Platyhelminthes: Seriata) tested with molecules and morphology. Molecular Phylogenetics and Evolution 2000;16:449-66.

[6] Hall T.A. (1999) BioEdit: a user-friendly biological sequence alignment editor and analysis program for Windows 95/98/NT, Nucleic Acids Symp. Ser. 41: 95-98.

[7] Kumar S., Stecher G., and Tamura K. (2016). MEGA7: Molecular Evolutionary Genetics Analysis version 7.0 for bigger datasets. Molecular Biology and Evolution 33:1870-1874. 